\title{
Simulation and FEM Analysis of Applied Force Effects for Energy Harvesting in Piezoelectric Bimorph Device
}

\author{
Panapong Songsukthawan ${ }^{\mathrm{a}}$, Chaiyan Jettanasen ${ }^{\mathrm{a},{ }^{*}}$ \\ ${ }^{a}$ Department of Electrical Engineering, Faculty of Engineering, \\ King Mongkut's Institute of Technology Ladkrabang, Bangkok 10520, Thailand \\ *kjchaiya@kmitl.ac.th
}

\begin{abstract}
Using of clean or renewable energy to generate electric power instead of fossil fuel is further promising. Smart material which can collect the dissipated vibration energy from moving object and transfer to electrical power called "Piezoelectric" material has developed and become increasingly interested. There are many kinds of synthetic material having piezoelectric effect; each type has different electrical and structural output when applying the same input. The structure analysis based on finite element method (FEM) is carried out in order to identify the optimized point of input force which will not damage the piezoelectric structure. The output voltage can be also determined. Moreover, the total displacement, equivalent stress and strain will be calculated and presented as contour mapping using ANSYS Workbench. The results are satisfactory and useful to identify material's characteristics appropriated to specific task and also reduce times and cost of trial and error phase in experiment.
\end{abstract}

Keywords: energy harvesting, finite element method, piezoelectric, PZT.

\section{Introduction}

Technological advancement needs a large quantity of electricity production which significantly rises up global warming gasses; environment and human being are directly affected and the hazardous effects have been daily increased. Alternative energy sources such as energy from the sun, wind, water, and geothermal are examples to generate electrical power as a clean and renewable ways instead of using fossil fuels.

It is highly interesting if we can take advantage from the energy dissipated within the ambience. Furthermore, if those energies are able to be accumulated for use in time of power shortage, it will be even more brilliant. The abovementioned principle is feasible using concept called electric energy harvesting from piezoelectric material. Piezoelectric material functions as generator, it converts motion and force to electric charge and voltage with several advantages, for instance, less complexity wiring and installation, great compatibility with other electronic devices and capability of operating without an external power supply [1]. The electrical characteristics described by the simulation result from MATLAB Simulink to indicate the harvested electrical energy in three configurations; single PZT, 2 PZTS in series and 2 PZTs in parallel, the voltage waveform will be presented in next section, and structural analysis and voltage intensity described by ANSYS Workbench based on finite element method confirm the possibility of exploiting these smart materials for power generation.

\section{Piezoelectricity}

\subsection{Characteristics}

Piezoelectric effect is property existing in many materials as it can transduce the mechanical action, whether compressive or tensile force into electricity. On the other hand, it can be reversibly converted; these phenomena are called piezoelectricity or piezoelectric effects. It is divided into two major features [2]. 


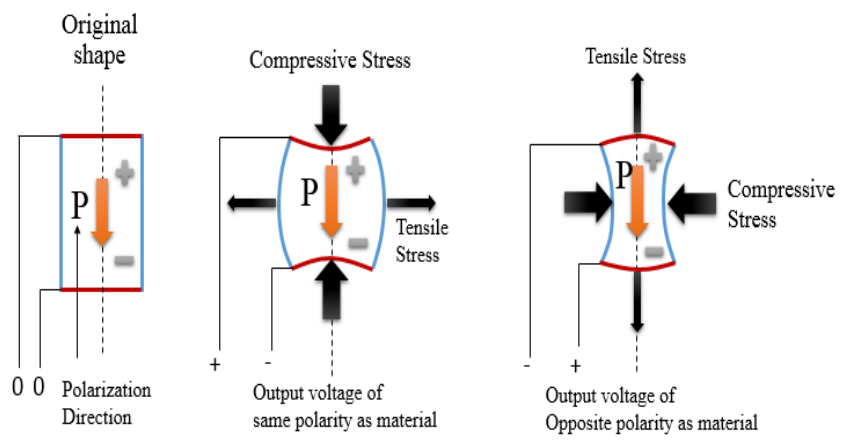

Fig.1. Direct piezoelectric effect.

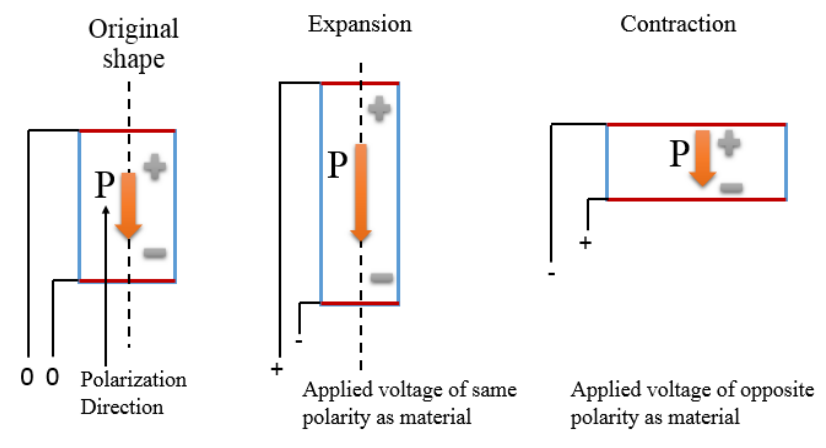

Fig.2 Converse piezoelectric effect

Direct piezoelectric effect is appearance of an electric potential across the crystal when it is received with mechanical forces, internal stress causes the ion arrangement, electric displacement formed, and then electric field is created, described by (1) and shown in Fig.1. Conversely, the application of a charge to the same material and become strained, this is proportional to the applied electric field, called converse piezoelectric effect described by (2) and shown in Fig.2.

Where,

$$
\begin{aligned}
& D_{i}=d_{i j} T_{j} \\
& S_{j}=d_{i j} E_{i}
\end{aligned}
$$

D: electric displacement vector or electric flux density

T: applied mechanical force

S: strain occurred within the crystal

E: electric field intensity vector applying to crystal

d: piezoelectric coefficient

These are all directional values; subscript " $i$ " represents an electrical direction ( $\mathrm{i}=1,2,3)$ and subscript ' $\mathrm{j}$ ' is related to mechanical direction $(\mathrm{j}=1,2,3, \ldots, 6)$, as illustrated in Fig.3. The digits marked in Fig. 3 are: 1, 2, 3 representing main axis, and 4, 5, 6 representing shear around the main axis.

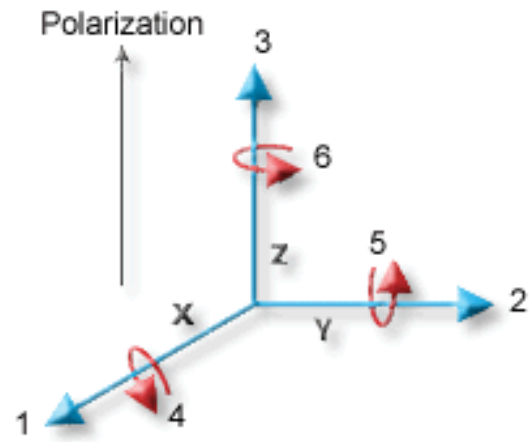

Fig.3. Directions of forces affecting a piezoelectric material.

Piezoelectric coefficient $\left(d_{i j}\right)$ are values related by two directions at the same time; they are very important parameters in energy harvesting. The harvested energy is proportional to these values. They also classify the operation modes of piezoelectric element, for example, $d_{33}$ is involved when the electric field is along the polarization axis (direction 3 ) and the mechanical strain is along the same axis and also called longitudinal effect or $d_{33}$ mode as shown in Fig.4, $d_{31}$ is applied if electric field is in the same direction as aforementioned and the strain is in orthogonal to the polarization axis (direction 1) and also called transverse effect or $d_{31}$ mode as shown in Fig.5
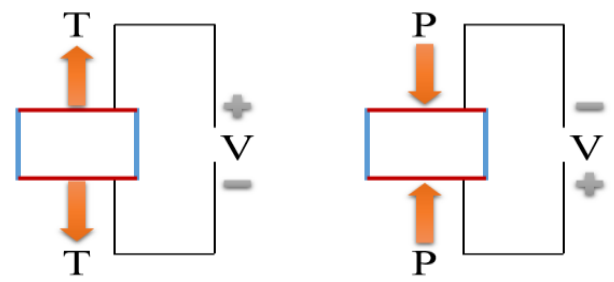

Fig.4. Longitudinal effect ( $d_{33}$ mode).

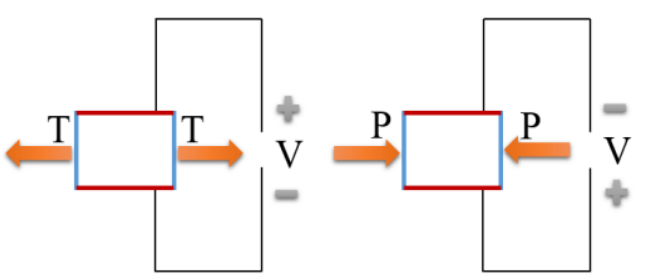

Fig.5. Transverse effect ( $d_{31}$ mode).

\subsection{Poling process}

The piezoelectric effect exhibited by naturally formed crystals such as Quartz, Topaz, Tourmaline, etc. and also other natural materials such as tendon, silk, dentin could not be directly used for energy harvesting applications. Consequently, the synthesized ceramics with randomly 
oriented grains have been developed with improved properties and aligning diploes by applying strong electric field across the material to exhibit piezoelectricity with poling process [3-4] as depicted in Fig.6

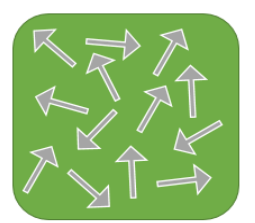

Random orientation of polar domains prior to polarization

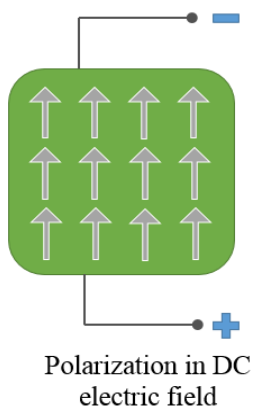

Fig.6. Poling process.

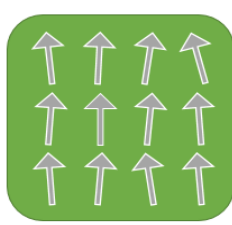

Remain polarization after electric field removed
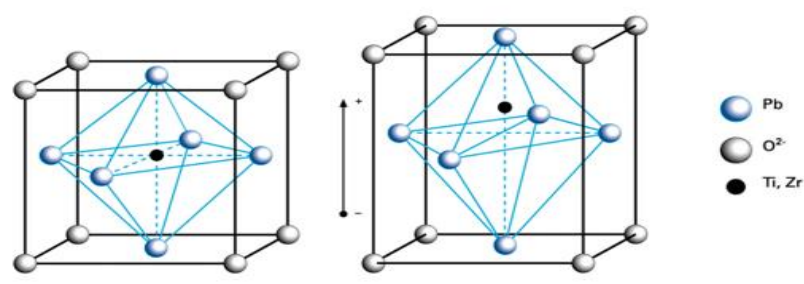

Fig.7. PZT and poled PZT structure.

\section{Simulation}

\subsection{Electrical simulation}

The chosen piezoelectric type is double-quick-mount bending generators, brass reinforcement enclosed by two layers of PZT-5A4E. When applying force, charge develops across each layers and accumulates in the brass substrate as the potential energy, until pausing applied force, it converts into kinetic energy and oscillates with its resonance frequency [5]. Fig.8 presents simulation schematic using MATLAB Simulink to indicate the electrical behavior with voltage waveforms given in Fig.9; there are the voltage results with setting input frequency of $5 \mathrm{~Hz}$, then illustrated with PZT configurations in series and in parallel. If using PZT in series configuration, the voltage is stepped up whereas if PZT sheets are set in parallel configuration, the voltage is unchanged, but the current is increased. shown in Fig.7

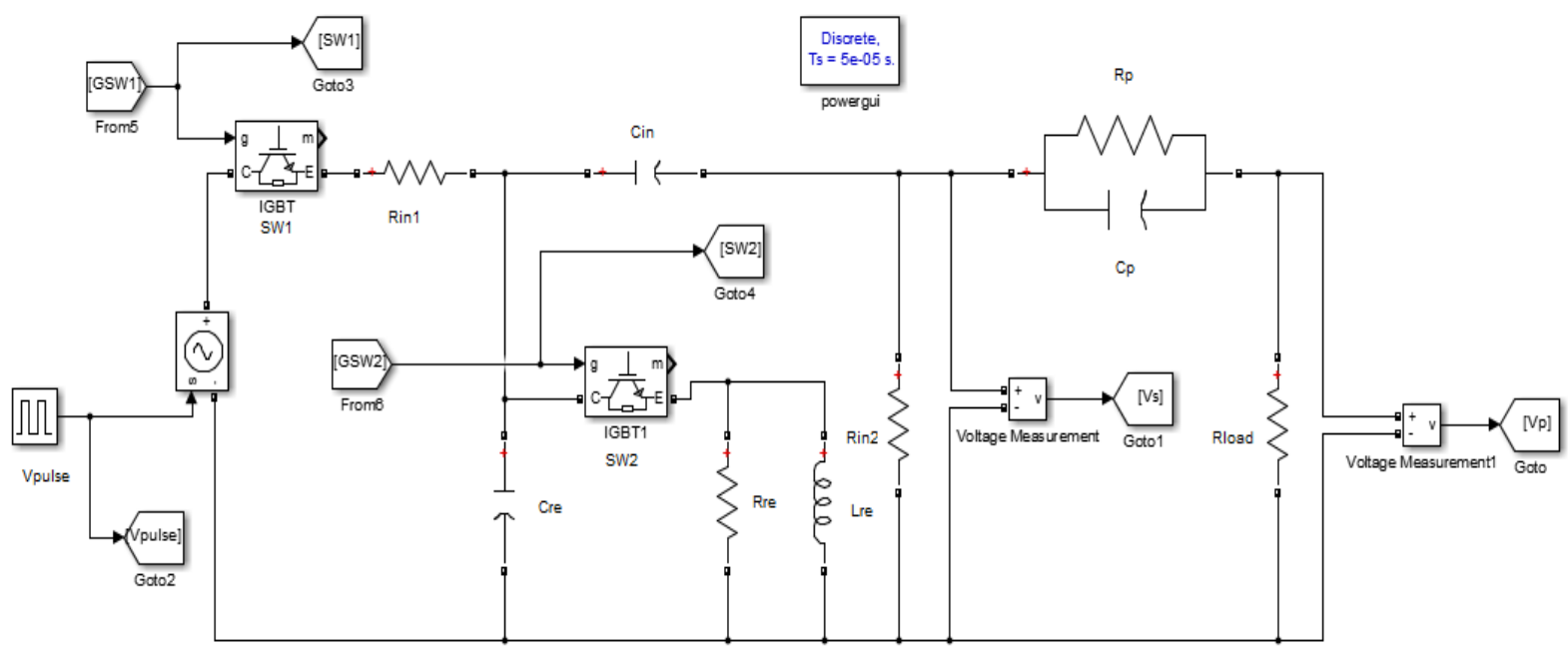

Fig.8. PZT schematics simulated by MATLAB Simulink. 

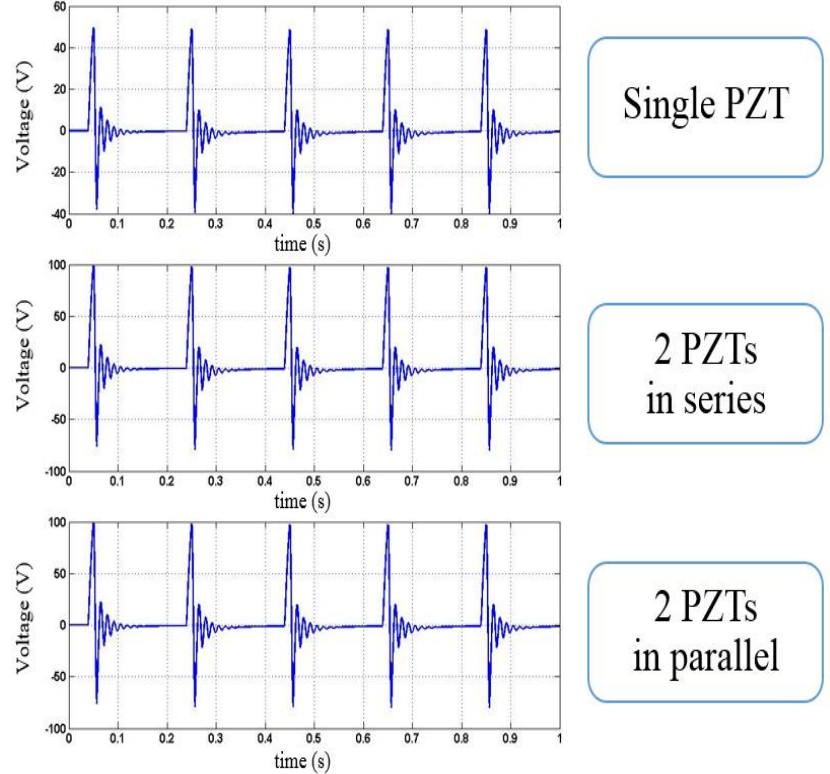

\section{PZTs \\ in parallel}

Fig.9. Voltage results for PZT in series and in parallel configurations.

\subsection{Structural simulation}

Even though PZT has a range of advantages, it is relatively brittle, and might be broken or damaged if applying too much force, and probably produces lower energy than expected level. Using finite element method helps in designing appropriate input (mechanical force or displacement) to achieve expected output voltage without the mechanical failure, and reduce the cost normally required in experiment or use with applications that is not yet fully tested, and most importantly, shorten trial and error times [6-8]. Fig.10 represents actual PZT structure and geometric model via ANSYS Workbench.
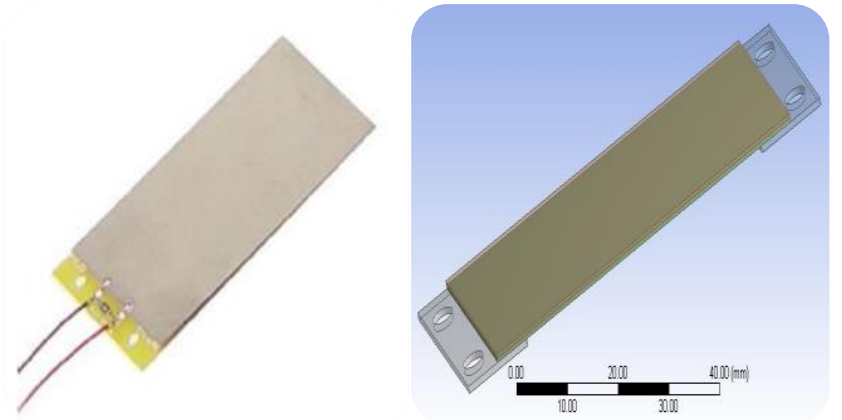

Fig.10. Actual PZT and geometric model.

In calculation process for piezoelectric device, material property data are typically provided as three matrices:

- Stiffness measured under constant electric field $\left[C_{E}\right]$
- Piezoelectric stress matrix $[e]$

- Relative permittivity measured under constant strain $\left[\varepsilon_{S}\right]$

The stiffness under constant electric field matrix $\left[C_{E}\right]$, computing compliance measured under constant electric field $\left[S_{E}\right]$ which each element is obtained by specific testing data of each piezoelectric material type and poled axis, then convert matrix's element from IEEE standard to ANSYS standard, for Y-poled, many of the constants in the matrix are equal to zero for the PZT piezoelectric ceramics, the non-zero constants are: $S_{11}^{E}$ value is placed at $S_{11}$ and $S_{33}, S_{33}^{E}$ value is placed at $S_{22}, S_{12}^{E}$ value is placed at $S_{13}$ and $S_{31}, S_{13}^{E}$ value is placed at $S_{23}$ and $S_{32}, S_{44}^{E}$ is placed at $S_{44}$ and $S_{55}$, and $S_{66}=$ $2\left(S_{11}-S_{12}\right)$, providing $\left[C_{E}\right]$ by (3)

$$
\left[C_{E}\right]=\left[S_{E}\right]^{-1}
$$

The piezoelectric stress matrix $[e]$, computing piezoelectric strain matrix $[d]$ by typical piezoelectric matrix, then transpose it and convert into ANSYS piezoelectric matrix; for Y-poled, Y-axis and Z-axis are rearranged, so $d_{31}$ value is put at $d_{12}$ and $d_{32}, d_{33}$ is put at $d_{22}$ and $d_{15}$ is put at $d_{41}$ and $d_{53}$, providing [e] by (4)

$$
[e]=\left[S_{E}\right]^{-1}[d]=[d]^{T}\left[S_{E}\right]^{-1}
$$

The relative permittivity measured under constant strain matrix $\left[\varepsilon_{S}\right]$, computing relative permittivity measured under constant stress $\left[\varepsilon_{T}\right]$, when $\left[\varepsilon_{T}\right]=\varepsilon_{0}\left[K_{T}\right]$, for Y-poled $K_{11}^{T}$ value is placed at $K_{11}$ and $K_{33}$ and $K_{33}^{T}$ value is placed at $K_{22}$, providing $\left[\varepsilon_{S}\right]$ by (5)

$$
\left[\varepsilon_{S}\right]=\left[\varepsilon_{T}\right]-[d]^{T}[e]
$$

The results are presented by contour mapping; the solutions consist of total deformation, equivalent elastic strain, and equivalent stress and voltage which are examples with the same distributed force in downward direction of 20 N, as depicted in Fig.11 to Fig.14.

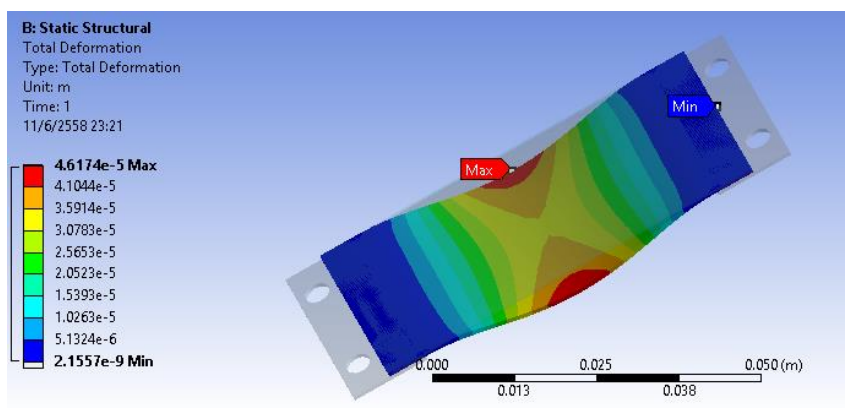

Fig.11. Total deformation contour result. 


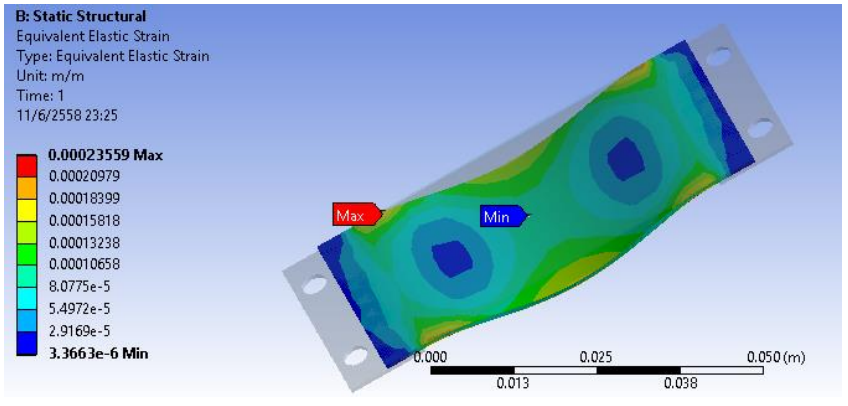

Fig.12. Equivalent elastic strain contour result.

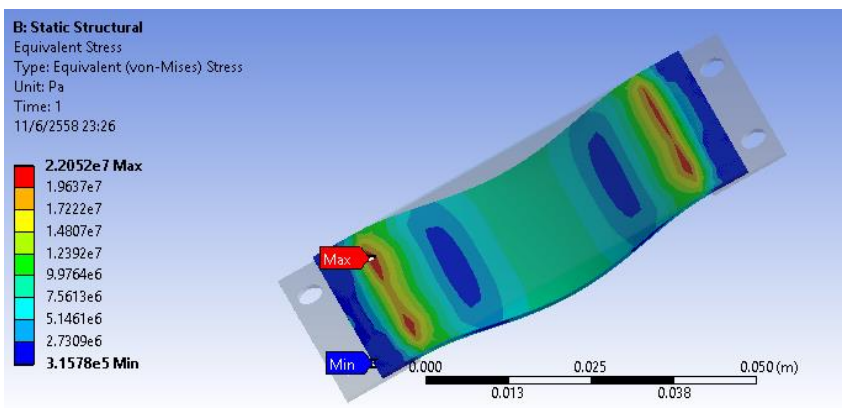

Fig.13. Equivalent stress contour result.

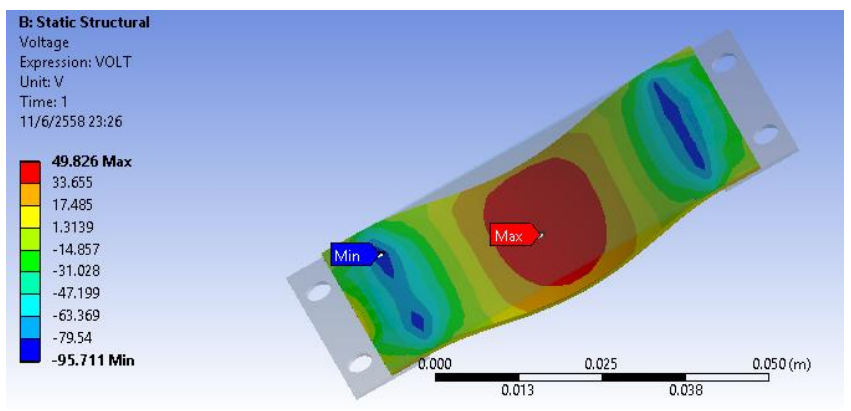

Fig.14. Voltage contour result.

\section{Conclusions}

This paper has presented the electrical characteristics and investigated structural analysis based on finite element method of piezoelectricceramic element PZT. The simulation is satisfactorily agreed. The electrical simulation by MATLAB Simulink showed that the voltage was increased when connecting PZT in series pattern and current was increased when connecting in parallel configuration. Furthermore, the structural analysis using ANSYS Workbench showed several results; total deformation to design direction and position of applied force, equivalent stress and strain to compare with relative yield strength of material in order to compute a safety factor and voltage result to meet a specific work or easily estimate expected voltage level from other piezoelectric material types.

\section{Acknowledgment}

The authors wish to gratefully acknowledge financial support for this research from the Research Fund of Faculty of Engineering, King Mongkut's Institute of Technology Ladkrabang, Thailand.

\section{References}

(1) D. Isarakorn, "Epitaxial Piezoelectric MEMS on Silicon" ECOLE POLYTECHNIQUE FEDERALE DE LAUSANNE EPFL, 2011.

(2) H. Kim, Y. Tedesse and S. Priya, "Energy Harvesting Technologies" , 2009.

(3) Dhananjay Kumar, Pradyumn Chaturvedi and Nupur Jejurikar, "Piezoelectric Energy Harvester Design and Power Conditioning”, IEEE Students' Conference on Electrical, Electronics and Computer Science (SCEECS) on 1-2 February 2014, pp. 1-6.

(4) Li Tianze, Zhang Xia, Jiang Chuan and Hou Luan, "Analysis of the Characteristics of Piezoelectric Sensor and Research of Its Application", 18th IEEE International Symposium on the Applications of Ferroelectrics on 23-27 August 2009, pp. 1-4.

(5) Uchino K. (2010). Advanced piezoelectric materials Science and technology. (1st Ed.) : Woodhead.

(6) Zhu Xiaojin; Zhao Miao; Gao Zhiyuan; Chen Zhiyan, "Analysis of active vibration control for piezoelectric intelligent structures by ANSYS and MATLAB," International Conference on Computer Application and System Modeling (ICCASM) 2010, vol.4, pp.V4184,V4-188, 22-24 Oct. 2010.

(7) Xiu-Mei Wang; Ju-Zheng Wang; Zhi-Yuan Gao; XiaoJin Zhu, "Simulation and experimental study on active vibration control of piezoelectric smart plate based on ANSYS," 2nd International Conference on Advanced Computer Control (ICACC), 2010, vol.1, pp.257-260, 27-29 March 2010.

(8) Chang Zheng; Xiao-ran Yin; Guo-qing Li; Xin-Yuan Miao, "Vibration control simulation of beams with piezoelectric actuators using impedance and FEM," Symposium on Piezoelectricity, Acoustic Waves and Device Applications (SPAWDA) 2011, pp.585-588, 911 Dec 2011. 